\title{
Réflexions sur femmes et propriété en France Évolutions récentes
}

\author{
Sylvette Denèfle \\ CITERES (Cités, Territoires, Environnement et Sociétés), UMR 7324, Université \\ de Tours \\ sylvabelle13@gmail.com
}

\begin{abstract}
As is the case for most of the legislation in France, the laws on property as well as those on inheritance do not differentiate between women and men. Nevertheless, several recent studies have revealed a disparity in the holding of assets between women and men, which fluctuate between 15 and 25 percent, whatever the social category. In statistical terms, women are less well off than men and, moreover, they are less often owners of their dwellings than men.

This contribution studies the inequalities between women and men in terms of property holding. It also analyzes forms of resistance within this context and, more specifically, types of resilience that women have developed today in order to overcome these circumstances, striving for a more egalitarian situation.

Property holding, as a principle which legitimizes equality, allows us to see that social practices, when studied through the lens of gender, reveal societies that are in reality very unequal and unfavorable towards women. This angle of study also brings to the fore the innovative resilience of women who reclaim an active part in citizenship and who no longer give in so easily to demands formulated in the past concerning their assigned social roles.
\end{abstract}

\section{Keywords}

property - assets - disparity in women and men - citizenship - resistance 


\section{Résumé}

Comme pour la plupart de la législation en France, les lois sur la propriété, de même que celles sur l'héritage, ne différencient pas les femmes et les hommes. Pourtant, plusieurs études récentes ont mis en évidence un écart de patrimoine variant entre 15 et $25 \%$, entre les femmes et les hommes, quelles que soient les catégories sociales. Les femmes se trouvent statistiquement moins aisées et, notamment, moins souvent propriétaires de leur logement que les hommes.

Ce texte propose d'éclairer ces inégalités entre les femmes et les hommes dans la propriété puis de présenter des formes de résistance, voire de résilience que les femmes d'aujourd'hui développent pour parvenir à des situations plus égalitaires.

La propriété en tant que principe légitimant l'égalité, permet de voir que les pratiques sociales, lorsqu'on les éclaire par la dimension du genre, présentent en fait des sociétés très inégalitaires qui ne sont guère favorables aux femmes. Mais elle éclaire aussi les résiliences innovantes des femmes qui revendiquent une citoyenneté active et ne se plient plus aussi facilement que dans le passé aux exigences de leurs assignations sociales.

\section{Mots clés}

propriété - patrimoine - écart femmes/hommes - citoyenneté - résistances

La propriété privée de l'habitation est au centre des valeurs qui ont construit la société française. Elle est associée à la citoyenneté, à l'identité locale et nationale, à l'établissement social, voire à la notabilité, à la vie familiale, à l'organisation de l'espace et à bien d'autres conceptions qui sont au cœur de la vie républicaine depuis la fin de l'Ancien Régime. A l'heure actuelle, elle reste une ambition sociale très largement partagée.

Comme pour la plupart de la législation en France, les lois sur la propriété, de même que celles sur l'héritage, ne différencient pas les femmes et les hommes. On pourrait donc penser qu'il n'y a pas ou très peu de différence entre les niveaux de propriété des unes et des autres. Il est d'ailleurs tout à fait notable que les études économiques, voire socio-économiques qui concernent le patrimoine des Français prennent très rarement en compte une variation entre les genres. 
Pourtant, plusieurs études récentes ${ }^{1}$ qui ont pris cette perspective ont mis en évidence un écart important de patrimoine entre les femmes et les hommes en France. Cet écart varie, selon les aspects considérés, entre 15 et $25 \%$, quelles que soient les catégories sociales. Les femmes se trouvent statistiquement moins aisées et, notamment, moins souvent propriétaires de leur logement que les hommes. Les raisons de cet écart sont multiples.

Tout d'abord, nous rappellerons ce que signifie la propriété et comment elle a évolué depuis le XVIII ${ }^{\mathrm{e}}$ siècle en France. Puis, nous verrons quelles explications peuvent nous permettre de comprendre les inégalités entre les femmes et les hommes dans la propriété. Enfin, nous examinerons, dans un troisième temps, les façons de vivre et les stratégies que les femmes déploient pour pallier à leurs difficultés économiques et pour affirmer leur citoyenneté à part entière, dans les années récentes. Nous présenterons des formes de résistance, voire de résilience qu'elles mettent en œuvre pour parvenir à des situations plus égalitaires.

\section{La propriété, au fondement de la citoyenneté française}

La propriété organise et stabilise les institutions sociales quelles que soient les cultures que l'on considère. Le plus souvent, c'est son caractère collectif ou individuel (notamment en ce qui concerne le foncier) qui détermine les types de société, et toujours elle modèle ou elle reflète, selon le point de vue que l'on adopte, les structures familiales (claniques, patri- ou matrilinéaire, personnelles, etc.). Elle constitue donc un vecteur fondamental des rapports entre les êtres humains. En France, le droit de la propriété a évolué au fil des siècles et s'est différencié selon les grands moments culturels. Aux pratiques des tribus gauloises, s'est imposé le droit romain qui a été remodelé par la féodalité avant que le XVIII ${ }^{\mathrm{e}}$ siècle révolutionnaire n'abolisse l'organisation tri-ordinale de l'Ancien Régime².

Les droits inégalitaires d'Ancien Régime sur le foncier ont généré les révoltes du XVIII ${ }^{\mathrm{e}}$ siècle à tel point que le droit de propriété a pu être considéré comme le symbole de l'égalité républicaine. La Déclaration des Droits de l'Homme et du Citoyen de 1789 pose dans son article ${ }^{\circ} 17$ que « La propriété étant un droit inviolable et sacré, nul ne peut en être privé » ; toutes les

\footnotetext{
1 Voir Patrimoine des ménages: nouveau contexte, nouveaux enjeux, Economie et Statistique INSEE (Institut national de la statistique et des études économiques), $\mathrm{n}^{\circ} 472-473$, décembre 2014.

2 Furet, 1988.
} 
constitutions françaisesquilui ont succédéont repris ceprincipepratiquementà l'identique ${ }^{3}$. Car c'est, en effet, la citoyenneté qui est déterminée de cette façon à travers l'Egalité, la Liberté et la Fraternité républicaines.

Pour ce qui est de la propriété privée, le principe en a été fondé par la République et n'a guère connu d'évolutions depuis la Révolution. C'est un droit sacré qui «prouve l'égalité possible entre les hommes, assure la liberté à laquelle tout un chacun aspire, fonde la dynamique économique capitaliste et structure profondément les territoires $»^{4}$.

Notre présent s'est construit dans la référence essentielle à la République, même si l'on peut encore trouver quelques traces de pratiques antérieures ayant quelque validité. C'est principalement le Code Civil de 1804 qui règle nos conceptions de la propriété, dans sa pérennité ou par ses remises en cause. Ce droit fondamental s'appuie sur le tryptique juridique «Usus, Fructus, Abusus » qui en détermine les contours 5 .

Ce qui nous intéresse particulièrement ici, c'est le lien étroit entre propriété et citoyenneté qui, dans la société «égalitariste» républicaine a exclu, selon les époques, les gens de couleur (encore esclaves au début du XIX ${ }^{\mathrm{e}}$ siècle), les populations autochtones de la colonisation et bien évidemment les femmes. La citoyenneté n'est véritablement accordée aux femmes qu'en 1946 et, si bien sûr, elles disposaient du droit de propriété, ce droit ne leur ouvrait ni la voie de l'égalité ni celle de la liberté. Les intellectuels et les militants ont toujours repensé et contesté la propriété mais leurs voix sont restées à la marge des pratiques socio-politiques dominantes ${ }^{6}$.

Pour l'ensemble du droit français, les conquêtes légales égalitaires entre les femmes et les hommes ont été achevées durant la seconde moitié du $\mathrm{Xx}^{\mathrm{e}}$ siècle mais on verra dans la suite qu'elles restent pourtant encore à mettre en pratique pour que les modes de vie s'accordent à la Loi écrite.

La société française, en construisant les assignations de rôles de sexe, notamment à travers les modèles familiaux, a toujours dérogé aux grands principes républicains et il est difficile de ne pas constater que notre présent est encore un héritage teinté de ces différences, ce que la propriété nous permet de lire de façon pertinente aussi bien dans sa pérennité que dans les contestations qu'elle rencontre au XXI ${ }^{\mathrm{e}}$ siècle.

3 Halpérin, 2008.

4 Denèfle, 2016, p. 9.

5 Denèfle, 2016 et https://fr.wikipedia.org/wiki/droit-de-propriete-en-france.

6 Josserand, 2006 ; Zenati, 1981. 


\section{$2 \quad$ Les femmes sont plus pauvres que les hommes}

Le $\mathrm{XX}^{\mathrm{e}}$ siècle a porté les revendications des femmes françaises à l'égalité, à travers la scolarisation, le travail, les droits familiaux, etc., et l'on peut avoir le sentiment d'un aboutissement législatif sur ces questions. Pourtant, les différences, voire les discriminations, entre les sexes, qui caractérisent nos pratiques sociales actuelles, demeurent vivaces et les travaux qui en analysent les diversités sont nombreux. Cependant, les études sur la propriété avec la perspective du genre sont relativement rares et sont récentes, alors qu'on a montré, par contre, depuis plusieurs décennies que les niveaux de revenus des femmes, principalement issus du salaire, étaient beaucoup plus faibles que ceux des hommes. De même, on a largement démontré que les retraites des femmes étaient très inférieures à celles des hommes. On sait aussi que les familles monoparentales sont à $85 \%$ féminines et que les métiers les plus fréquemment occupés par des femmes sont parmi les moins bien rémunérés. Ces constats, maintenant passés dans la connaissance commune ${ }^{7}$, donnent lieu à des politiques spécifiques qui tendent à corriger les inégalités sociales. Il n'en demeure pas moins que les femmes sont plus pauvres que les hommes, que cela soit parce qu'elles sont plus nombreuses dans des positions sociales défavorisées ou par le fait d'inégalités de revenus et de patrimoine, quelles que soient les catégories sociales ${ }^{8}$. Ces constats, issus d'études fines, d'une inégalité de fait des femmes heurtent le sens commun, politiquement très égalitariste en France. Mais ils résistent à l'examen.

En effet, si nous considérons les évolutions législatives dans un parallèle avec celles des pratiques sociales, depuis le milieu du $\mathrm{Xx}^{\mathrm{e}}$ siècle, nous notons tout d'abord, une entrée massive des filles dans le système scolaire. Alors qu'elles étaient très minoritaires dans l'enseignement secondaire après la Deuxième Guerre mondiale, elles sont devenues progressivement majoritaires pour l'obtention du baccalauréat à la fin des années $70^{9}$. C'est à partir du début des années 80 que les filles sont devenues majoritaires dans l'enseignement supérieur pour en atteindre $60 \%$ des effectifs à l'heure actuelle.

Dans l'ordre du travail salarié, la France a connu des évolutions comparables. Les années 60 constituent un moment d'étiage du salariat féminin. Selon l'enquête emploi de l'inseE (Institut national de la statistique et des

7 Tableaux de l'économie française, INSE E, 02/03/2017, voir www.insee.fr.

8 Ainsi, il n'y a guère de travaux effectués dans le cadre du groupe de recherche MAGE (Marché du Travail et Genre) qui n'analyse finement les mécanismes des discriminations économiques touchant les femmes.

9 Baudelot et Establet, 1989. 
études économiques) de 1981, dans les années 6o, moins de $30 \%$ des femmes en âge de travailler étaient salariées ${ }^{10}$ et un tiers des femmes en âge de travailler, étaient encore inactives au début des années 8o. Elles représentaient alors un tiers des actifs ${ }^{11}$. Selon Cédric Afsa Essafi et Sophie Buffeteau ${ }^{12}$, dans la première décennie du xxI ${ }^{\mathrm{e}}$ siècle, les trois-quarts des femmes, en âge de le faire, travaillent et elles représentent pratiquement la moitié des actifs.

On pourrait donc penser que les inégalités constatées entre les femmes et les hommes concernent le passé ou du moins sont en voie de résolution, comme le voudrait le sens commun.

Mais cela n'est pas le cas, et ne le sera pas dans un avenir proche puisque, d'une part, les femmes sont scolarisées dans des filières de professionnalisation moins porteuses sur le marché du travail que les hommes pour les actifs diplômés et, d'autre part, l'écrasante majorité des femmes qui sont salariées le sont dans des secteurs d'emploi très défavorables. Le taux de chômage des femmes est supérieur d'un tiers à celui des hommes et les femmes sont $32 \%$ à occuper un emploi à temps partiel pour $6 \%$ des hommes. Par ailleurs, le salariat féminin est centré sur un très faible nombre d'emplois qui sont des emplois de faible qualification et à basse rémunération.

Ces données structurelles très connues de l'emploi féminin permettent dans un premier temps d'envisager là quelques causes des inégalités de revenus entre les femmes et les hommes. Mais il faut y rajouter l'incompressible différence de niveau de rémunération, pratiquement tous emplois confondus, dont la seule cause semble être le genre. Les femmes cadres touchent à emploi équivalentenviron $25 \%$ de moins que leurs collègues masculins, c'est $15 \%$ de moins pour les ouvriers ou les professions intermédiaires et moins de $10 \%$ pour les employés (secteur d'activité où les femmes représentent l'écrasante majorité des travailleurs). A ces écarts de traitement, on ne peut oublier d'ajouter les différences de carrière entre les femmes et les hommes qui cantonnent les femmes à des niveaux de promotion très inférieurs à ceux des hommes. Enfin, les secteurs d'emploi des services qui sont ceux qui produisent le plus grand nombre de travail à temps partiel et les rémunérations les plus faibles (femmes de ménage, aides aux personnes, garde d'enfants, etc.) sont occupés à plus de $80 \%$ par des femmes ${ }^{13}$.

\footnotetext{
$10 \quad$ Levy et Labourie-Racape, janvier 1983.

11 Maruani, 2004.

12 Afsa Essafi et Buffeteau, 2006.

13 Pour l'ensemble de ces données économiques concernant le travail, voir Tableaux de l'économie française, INSEE, 02/03/2017, www.insee.fr.
} 
Clairement donc, si l'on considère les ressources économiques liées au travail dont disposent les femmes et les hommes, on note un incontestable écart entre les genres. Les raisons en sont très diverses mais elles participent toutes des modèles sociaux de genre qui fondent l'organisation sociale en France. L'une de ces raisons est particulièrement importante : l'organisation familiale. Dans la famille, les femmes ont la charge du travail domestique et cette charge n'est pas partagée lorsque les femmes travaillent. Elle se surajoute à leurs activités. Parmi le travail domestique, l'éducation des enfants est une partie considérable des activités féminines. Sans entrer dans les pratiques de chacun, on peut noter un indicateur intéressant de cette assignation des femmes à la famille, celui de la monoparentalité qui est à près de $90 \%$ féminine ${ }^{14}$.

A l'évidence, les familles monoparentales, parce qu'elles ne disposent que d'un revenu, lorsque c'est le cas, et parce qu'elles ne peuvent en disposer dans un grand nombre de situations, constituent des noyaux de pauvreté. Il n'est que de constater que dans certains quartiers d'habitat social, les logements aidés sont occupés jusqu'à près de $40 \%$ par des femmes en situation de monoparentalité, pour mesurer les causes structurelles des inégalités économiques entre les femmes et les hommes.

A l'autre bout de l'échelle des âges, la situation des femmes n'est pas meilleure. En effet, parce qu'elles ont eu des carrières commencées tardivement, dans des secteurs d'emploi peu favorables, les retraites des femmes sont très inférieures à celles des hommes (un rapport de juin 2015 du Conseil d'Orientation des Retraites estime qu'en moyenne les retraites des femmes sont égales à $68 \%$ de celles des hommes).

Il est donc manifeste que sous l'aspect des revenus du travail, les femmes sont dans des situations beaucoup moins favorables que les hommes et que de surcroît, elles sont très majoritaires dans les contextes de grande, voire très grande pauvreté.

D'autre part, les contextes socio-culturels, d'une certaine façon, n'engagent pas les femmes dans la voie de la propriété du logement car la société répugne à laisser à la rue les familles avec des enfants, et donc les femmes, avec des enfants ou non, dans la mesure où elles sont socialement désignées comme victimes potentielles de toutes sortes de malveillances. La logique sociale, protectrice des femmes supposées faibles, est donc une ressource pour celles qui en ont besoin et ne les engage pas à des initiatives plus autonomes.

Curieusement, cet héritage social du moindre dynamisme économique des femmes est mobilisé également comme significatif par les économistes qui

14 «Les familles monoparentales : des difficultés à travailler et à se loger », Insee Première, INSEE, $\mathrm{n}^{\circ} 1195,01 / 06 / 2008$. 
constatent que, hors du champ salarial, les patrimoines féminins sont toujours statistiquement inférieurs à ceux des hommes ${ }^{15}$. Ce type d'étude est extrêmement récent ${ }^{16}$ et n'avait jamais retenu l'attention des chercheurs, notamment parce que les données permettant de le documenter sont difficiles à mobiliser, l'habitude étant de traiter du patrimoine des ménages et non de celui des individus le composant. Pourtant, ces études permettent de voir que, dans les patrimoines mobiliers et immobiliers, dans les patrimoines financiers, les femmes qui, dans ces contextes ne sont guère susceptibles de très grande difficulté sociale, se trouvent néanmoins dans des positions d'inégalité, et cela nonobstant la forte homogamie sociale. Ces inégalités qui peuvent aller, selon les variables que l'on considère, de 5 à $40 \%$, ont des origines complexes qui sont liées aux ressources du travail, à celles de l'héritage, à celles des pratiques d'accumulation du capital ${ }^{17}$. Elles proviennent de surcroît de l'intériorisation des valeurs sociales dominantes sur la place de la maternité ou celle du pouvoir. Il est intéressant de noter que ces inégalités perdurent, même avec la prise en compte du veuvage dont on sait qu'il est très majoritairement féminin, donc potentiellement favorable au patrimoine féminin. D'ailleurs, si l'on compare les patrimoines des veuves de plus de 65 ans et celui des couples du même âge, on note que les veuves ont 2,5 fois moins de patrimoine que les couples et qu'elles sont deux fois moins propriétaires de leur résidence principale ${ }^{18}$ que les couples.

En moyenne, les écarts entre femmes et hommes, tous âges confondus, sont plus faibles pour ce qui concerne le patrimoine immobilier que ceux qui concernent le patrimoine financier car, entre autres, pour une part importante de la population, la propriété de l'habitation principale d'une famille est détenue par le couple (84\%). Mais on se rappellera également qu'une $\operatorname{loi}^{19}$ a dû contraindre les entreprises importantes à accorder un droit de participation aux femmes dans les conseils d'administration et que les grandes directions restent essentiellement masculines, les figures féminines faisant exception.

\footnotetext{
15 Bonnet, Keogh et Rapoport, 2014. Ces auteurs utilisent l'enquête Patrimoine de l'Insee de 2004 et 2010.

16 Par exemple, Pikety, 2013, qui s'intéresse au patrimoine du millième le plus riche de la population.

17 « Patrimoine des ménages : nouveau contexte, nouveaux enjeux », Economie et Statistique, INSEE, $\mathrm{n}^{\circ}$ 472-473, décembre 2014.

18 Lorsqu'elles sont propriétaires de leur résidence principale, celle-ci a une valeur deux fois moins élevée que celle des couples (Voir le $6^{\mathrm{e}}$ rapport annuel du Conseil d'Orientation des Retraites, 2008).

19 Loi Copé-Zimmermann de 2011.
} 
L'ensemble de ces données montre donc largement que les femmes, quelle que soit la catégorie sociale que l'on considère, sont dans des situations économiques moins favorables que les hommes, et cela à la fois pour des raisons structurelles (fait générationnel, type d'emploi, type de formation, etc.) et pour des raisons culturelles (valeurs maternelles, modes familiaux, rapport au pouvoir, etc.). Il est manifeste que le retentissement en est important sur l'accès à la propriété.

\section{Les femmes déploient des formes de résistance}

Les économistes ont montré que les évolutions récentes tendaient à réduire les écarts entre femmes et hommes mais dans une mesure très faible qui laisse des écarts pour lesquels les explications sont difficiles à apporter. Cela doit nous alerter sur les difficultés à réaliser l'égalité. Mais pourtant, les femmes s'essaient à faire évoluer leurs situations.

Des faits sociaux récents peuvent nous permettre de mettre en évidence des changements conséquents dans les comportements féminins dont on peut penser qu'ils modifieront les rapports à la propriété, aux ressources pour d'autres modes de vie plus égalitaires ou plus solidaires.

Ces évolutions concernent, pour une part, l'ensemble social et sous d'autres aspects, elles sont plus spécifiquement féminines.

Dans un ouvrage récent, nous avons montré qu'alors qu'elle est fortement corrélée au développement économique du monde occidental, toute remise en cause de la propriété privée est perçue comme « une destruction des bases de la société conduisant à un chaos inévitable ou à un totalitarisme inacceptable ${ }^{20}$. Cependant, les difficultés que connaît actuellement le modèle socio-économique et politique de l'Occident (crises boursières, crises écologiques, crises militaires, voire religieuses, migratoires, etc.), amènent une partie de la population, notamment la jeunesse scolarisée à douter de l'idéologie du progrès qui a fondé le développement par la croissance après la Seconde Guerre mondiale. C'est, par contrecoup, la propriété qui est déstabilisée et, tout particulièrement, la propriété du logement qui était symboliquement associée à l'accès pour tous aux richesses et à l'égalité dans les années 70. Au XXI ${ }^{\mathrm{e}}$ siècle, une partie importante des classes moyennes disposant d'un capital culturel relativement important, se trouve dans l'impossibilité d'accéder à la propriété du logement par l'enchérissement démesuré du foncier et de l'immobilier dans les centres urbains. La reconsidération du sens de la propriété privée individuelle 
conduit de plus en plus à des logiques de mutualisation, de partage, de mise en commun des compétences et des ressources qui déportent la propriété privée vers la propriété collective. Par ailleurs, les évolutions technologiques qui mettent sur le marché des biens en abonnement et non plus en propriété de la chose (téléphone, internet, etc.) induisent des comportements d'utilisation qui conduisent vers la propriété d'usage. Enfin, les formes de plus en plus importantes de services pour l'accès à des biens (location/partage de voitures, de logement, d'outils, de services, etc.) font glisser la propriété vers des formes de propriété temporaire qui minent l'idéologie juridique traditionnelle.

A l'heure actuelle, assez logiquement du fait de ces évolutions, on voit émerger des reconsidérations sur la propriété, comme propriété d'usage ou comme propriété partagée, comme propriété temporaire ou en jouissance qui parfois recoupent la propriété en biens communs et qui démembrent le droit de la propriété en mettant l'accent sur ses fonctions. Ces évolutions sont parfois étroitement liées aux évolutions sociales les plus courantes. On le voit notamment sur les changements de pratiques des personnes âgées, et bien évidemment des femmes âgées qui, avec des revenus modestes, ont l'inquiétude des services qui leur seront nécessaires avec une arrivée possible de la dépendance et préfèrent renoncer à la transmission d'un héritage modeste à leur descendance pour assurer un ensemble de services de fin de vie dans un habitat coopératif ou dans un logement collectif.

L'ensemble des changements de nos pratiques sociales récentes, en matière de propriété, et notamment de propriété du logement, trouvent un écho tout à fait spécifique pour les femmes. En effet, le fait de vieillissement est démographiquement plus féminin que masculin et l'on a vu que les ressources des femmes âgées sont nettement plus faibles que celles des hommes. C'est l'une des raisons qui conduit bien des femmes âgées à des pratiques de location d'une partie de leur habitation à des jeunes qui sont, pour le cas général, plutôt des jeunes femmes ${ }^{21}$. Ici encore, les considérations sur la faiblesse des femmes les conduit à chercher à travers leur genre un soutien et une sécurité qui est moins évidente pour les hommes.

Lorsqu'elles sont propriétaires de leur logement, il arrive également qu'elles connaissent des difficultés à l'entretenir ou à en assumer les charges. Il en résulte un intérêt très marqué des femmes pour les expérimentations d'habitat participatif où le partage de services et d'espaces est la règle. L'exemple très connu de la maison des Babayagas de Montreuil ${ }^{22}$ en est une illustration

\footnotetext{
21 Pennec, 2016, p. 127.

22 Voir http://www.histoiresordinaires.fr/Therese-clerc-87-ans-Le-voyage-a-ete-si-beaua1612.html.
} 
manifeste. Mais les enquêtes sur le développement de cette forme d'habitat, partout dans le monde, amènent au même constat. Les femmes sont majoritaires dans les expériences d'habitat participatif. De la même façon, on note que toutes les expériences connues de maisons communes pour les familles monoparentales réunissent essentiellement des femmes qui y trouvent des aides et des soutiens, dans la vie familiale et domestique mais aussi pour l'insertion professionnelle. Cette fois, c'est la monoparentalité quasi exclusivement féminine qui induit des formes de résilience spécifique dans la recherche de logements qui comportent des solutions à d'autres aspects de leurs difficultés sociales que la seule faiblesse de leurs ressources. Les solidarités féminines jouent alors de façon sensible.

En réponse aux difficultés d'accès au logement pour les jeunes adultes sans emploi, le développement des habitats mobiles ou précaires devient assez répandu en zone rurale. Ces pratiques concernent plus les familles que les femmes seules, du fait probablement de contraintes spécifiques pour la construction ou l'accès aux services. Mais dans les franges les plus militantes de ces populations, on trouve, de façon marginale mais exemplaire, des expériences de squats féministes qui semblent résumer les formes de résiliences militantes ${ }^{23}$. Elles tendent à redonner aux femmes des positions non discriminées où toutes les formes de pouvoir sont repensées et déconstruites en vue de faire évoluer l'ensemble social vers plus d'égalité entre les genres.

\section{Conclusion}

La lecture genrée de la propriété n'apparaît pas immédiatement pertinente au sens commun qui ne veut lire que les formes égalitaristes de la loi française. Mais cependant toutes les analyses montrent que la disparité est grande entre les hommes et les femmes à l'intérieur de chaque catégorie sociale. Malgré l'effacement de cette régularité par la perception des différences entre les catégories sociales, force est bien d'affirmer l'écart entre les femmes et les hommes en matière de propriété, comme c'est le cas pour l'ensemble des ressources sociales.

Ces différences que l'on peut lire en terme de discriminations de genre, lorsqu'on a épuisé toutes les autres explications, amènent à des remises en question des grands principes des sociétés qui les pratiquent, voire qui les légitiment ou à tout le moins qui les invisibilisent. C'est ainsi que les crises socio-économiques récentes provoquent des remises en question des démocraties représentatives

23 Gaillard, 2016, p. 201. 
qui ouvrent la porte à des formes spécifiques de résiliences féminines dans les situations tout à fait pragmatiques de leurs difficultés quotidiennes.

La propriété est un de ces principes qui, légitimant l'égalité, permet de voir que les pratiques sociales, lorsqu'on les éclaire par la dimension du genre, présentent en fait des sociétés très inégalitaires qui ne sont guère favorables aux femmes. Mais elle éclaire aussi les résiliences innovantes des femmes qui ne se plient plus aussi facilement que dans le passé aux exigences de leurs assignations sociales.

\section{Bio}

Professor emeritus of sociology, Sylvette Denèfle is a member of the CNRS "Cities, Territories, Environment and Societies" team. Within this context, she researches the question of social norms and, more particularly, ideologies, gender-related social relationships, and urban development. She was coordinator of several European and French research programs and networks. She was the head of different research teams as well as an international expert and member of several national and international scientific councils.

For more details and publications, see www.sylvette-denefle.com.

\section{Références bibliogaphiques}

Afsa Essafi C. et Buffeteau S., 2006, « L'activité féminine en France : quelles évolutions récentes, quelles tendances pour l'avenir?», Economie et Statistique $\mathrm{n}^{\circ} 39^{8-399}$, p. $85^{-97}$.

Baudelot C. et R. Establet, 1989, Le niveau monte, Paris, Seuil.

Bonnet C., A. Keogh et B. Rapoport, 2014, «Quels facteurs pour expliquer les écarts de patrimoine entre hommes et femmes en France?» Economie et Statistique $\mathrm{n}^{\circ} 472-473$, p. 101-123.

Denèfle S., dir., 2016, Repenser la propriété. Des alternatives pour habiter, Rennes, Presses Universitaires de Rennes (PUR).

Furet F., 1988, La Révolution 1770-1814, Paris, Hachette.

Gaillard E., 2016, « Les squats féministes : une lutte pour l'émancipation et l'autonomie » in S. Denèfle, Repenser la propriété. Des alternatives pour habiter, Rennes, PUR.

Halpérin, J. L., 2008, Histoire du droit des biens, Paris, Economica.

INSEE (Institut national de la statistique et des études économiques), 2017 (2 mars)

Tableaux de l'économie française, www.insee.fr. 
INSEE, 2014 (décembre), «Patrimoine des ménages: nouveau contexte, nouveaux enjeux », Economie et Statistique, $\mathrm{n}^{\circ} 472-473$.

INSEE, 2008 (1 juin), «Les familles monoparentales: des difficultés à travailler et à se loger $»$, Insee Première, $\mathrm{n}^{\circ} 1195$.

Josserand L., 2006 (ré-édition de 1939), De l'esprit des droits et de leur relativité, Paris, Dalloz.

Levy M. et A. Labourie-Racape, 1983 (janvier), « Le salariat féminin en perspectives », Population et Sociétés, $\mathrm{n}^{\circ}$ 165, p. 77-83.

Maruani M., 2004, «Activité, précarité, chômage : toujours plus?» in F. Milewski et H. Périvier, Travail des femmes et inégalités, Revue de l'OFCE (Observatoire français des conjonctures économiques), $\mathrm{n}^{\circ}$ 90, p. 95-115.

Pennec S., 2016, «La propriété questionnée par le vieillissement» in S. Denèfle Repenser la propriété. Des alternatives pour habiter, Rennes, PUR.

Pikety I., 2013, Le capital au XXIè siècle Les livres du nouveau monde, Paris, Seuil.

Zenati F., 1981, Essai sur la nature juridique de la propriété: Contribution à la théorie du droit subjectif, Université de Lyon, thèse de doctorat.

https://fr.wikipedia.org/wiki/droit-de-propriete-en-france

http://www.histoiresordinaires.fr/Therese-clerc-87-ans-Le-voyage-a-ete-si-beau-_ a1612.html 